\title{
Quantification of oxide particle composition in model oxide dispersion strengthened steel alloys
}

\author{
A.J. London ${ }^{\mathrm{a}, *}$, S. Lozano-Perez ${ }^{\mathrm{a}}$, M.P. Moody ${ }^{\mathrm{a}}$, S. Amirthapandian ${ }^{\mathrm{b}}$, B.K. Panigrahi ${ }^{\mathrm{b}}$, \\ C.S. Sundar ${ }^{b}$, C.R.M. Grovenor ${ }^{a}$ \\ a Department of Materials, University of Oxford, Parks Road, Oxford OX1 3PH, UK \\ ${ }^{\mathrm{b}}$ Indira Gandhi Centre for Atomic Research, Kalpakkam 603 102, TN, India
}

\section{A R T I C L E I N F O}

Article history:

Received 29 August 2014

Accepted 18 February 2015

\begin{abstract}
A B S T R A C T
Oxide dispersion strengthened ferritic steels (ODS) are being considered for structural components of future designs of fission and fusion reactors because of their impressive high-temperature mechanical properties and resistance to radiation damage, both of which arise from the nanoscale oxide particles they contain. Because of the critical importance of these nanoscale phases, significant research activity has been dedicated to analysing their precise size, shape and composition (Odette et al., Annu. Rev. Mater. Res. 38 (2008) 471-503 [1]; Miller et al., Mater. Sci. Technol. 29(10) (2013) 1174-1178 [2]).

As part of a project to develop new fuel cladding alloys in India, model ODS alloys have been produced with the compositions, $\mathrm{Fe}-0.3 \mathrm{Y}_{2} \mathrm{O}_{3}, \mathrm{Fe}-0.2 \mathrm{Ti}-0.3 \mathrm{Y}_{2} \mathrm{O}_{3}$ and $\mathrm{Fe}-14 \mathrm{Cr}-0.2 \mathrm{Ti}-0.3 \mathrm{Y}_{2} \mathrm{O}_{3}$. The oxide particles in these three model alloys have been studied by APT in their as-received state and following ion irradiation (as a proxy for neutron irradiation) at various temperatures. In order to adequately quantify the composition of the oxide clusters, several difficulties must be managed, including issues relating to the chemical identification (ranging and variable peak-overlaps); trajectory aberrations and chemical structure; and particle sizing. This paper presents how these issues can be addressed by the application of bespoke data analysis tools and correlative microscopy. A discussion follows concerning the achievable precision in these measurements, with reference to the fundamental limiting factors.
\end{abstract}

(c) 2015 Elsevier B.V. All rights reserved.

\section{Introduction}

To advance our understanding of the behaviour of oxide dispersion strengthened steels (ODS), accurate microstructural characterisation is required. The particle size distribution influences the mechanical properties [3] and understanding the chemistry and composition of these particles informs predictions of the alloy development during processing and in service [4]. Atom probe tomography (APT) has the spatial and chemical resolution required to resolve the smallest oxide clusters and to study their character as a function of thermal [5] and irradiation treatments [6]. Significant controversy has surrounded the characterisation of the oxide particles that reinforce ODS alloys [7-10], which may be in part due to the differences in alloy content, processing methods and techniques employed [11]. Composition measured by APT relies on the interpretation of the time-of-flight mass spectrum data but details are rarely reported on the identification of mass peaks and how potential peak overlaps are resolved, complicating

\footnotetext{
* Corresponding author.

E-mail address: andrew.london@materials.ox.ac.uk (A.J. London).
}

the comparison of results between different materials and groups. To investigate the origins of compositional discrepancies, a combination of APT analysis of model alloys with correlative microscopy (TEM and APT) and a systematic and cluster-by-cluster approach to composition measurement has been implemented.

Three model alloys have been produced, with target compositions of $\mathrm{Fe}-0.3 \mathrm{Y}_{2} \mathrm{O}_{3}, \mathrm{Fe}-0.2 \mathrm{Ti}-0.3 \mathrm{Y}_{2} \mathrm{O}_{3}$ and $\mathrm{Fe}-14 \mathrm{Cr}-0.2 \mathrm{Ti}-0.3 \mathrm{Y}_{2} \mathrm{O}_{3}$ (all wt\%), as part of a major Indian project to produce ODS nuclear fuel cladding tubes. These alloys represent an excellent opportunity to study model systems of well-known provenance, without the complications of the additional alloying elements in conventional ODS alloys. Even in these simple alloy systems, APT characterisation presents some challenges, including the quantification of oxide stoichiometry and their locally varying field-evaporation behaviour. We present our pragmatic approach to APT analysis of these materials and the methods used, acknowledging the sources of error in light of the limitations of APT.

\section{Materials and methods}

Three model ODS alloys were prepared by mechanical alloying 
and hot extrusion of elemental powders. High purity elemental powder mixtures of target compositions $\mathrm{Fe}-0.3 \mathrm{Y}_{2} \mathrm{O}_{3}$, $\mathrm{Fe}-0.2 \mathrm{Ti}-0.3 \mathrm{Y}_{2} \mathrm{O}_{3}$ and $\mathrm{Fe}-14 \mathrm{Cr}-0.2 \mathrm{Ti}-0.3 \mathrm{Y}_{2} \mathrm{O}_{3}$ (all wt\% and $<0.03 \mathrm{wt} \% \mathrm{C}$ ) were mechanically alloyed by ball milling (Simolayer CM-08, argon atmosphere, $4 \mathrm{~h}$ at $1000 \mathrm{rpm}, 10: 1$ ball to powder ratio). The mechanically alloyed powders, $\mathrm{Fe}-0.3 \mathrm{Y}_{2} \mathrm{O}_{3}$ and Fe-0.2Ti-0.3 $\mathrm{Y}_{2} \mathrm{O}_{3}$, were extruded at $1050{ }^{\circ} \mathrm{C}$ followed by annealing at $950{ }^{\circ} \mathrm{C}$ for $2 \mathrm{~h}$ and air cooling. The mechanically alloyed $\mathrm{Fe}-14 \mathrm{Cr}-0.2 \mathrm{Ti}-0.3 \mathrm{Y}_{2} \mathrm{O}_{3}$ powder was extruded at $1150{ }^{\circ} \mathrm{C}$, quenched and tempered at $750{ }^{\circ} \mathrm{C}$ for $2 \mathrm{~h}$ and air cooled. Consolidated ODS rods $12 \mathrm{~mm}$ in diameter were prepared from all three alloy compositions.

APT needles were made by conventional electropolishing methods [12] and by FIB lift-out [13]. TEM analysis was carried out using a Carl Zeiss LIBRA 200FE (microstructural observations), Philips CM20 (screening of APT needles before analysis) and a monochromated and double- $\mathrm{C}_{\mathrm{s}}$-corrected JEOL 2200MCO FEG microscope (EFTEM imaging; see [14] for details). Atom probe analyses were performed using a LEAP-3000X HR microscope at an analysis temperature of $50 \mathrm{~K}$. A $532 \mathrm{~nm}$ wavelength laser with a pulse width of $10 \mathrm{ps}$, repetition rate of $200 \mathrm{kHz}$, pulse energy of $0.3-0.4 \mathrm{~nJ}$ and spot size of less than $10 \mu \mathrm{m}$ was used to promote field evaporation. Oxide clusters in the data were identified using the method of maximum separation [15] of Y, YO, TiO and O ions. A 0.7-1.1 nm maximum separation of core ions was used with a $N_{\text {min }}$ value between 8 and 15 by the method explained in [16].

a

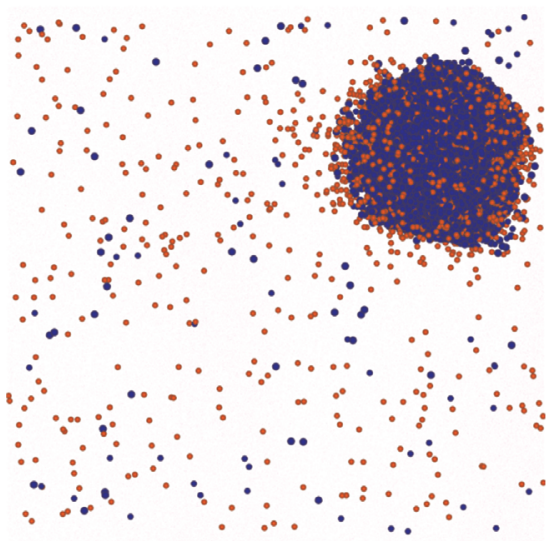

C

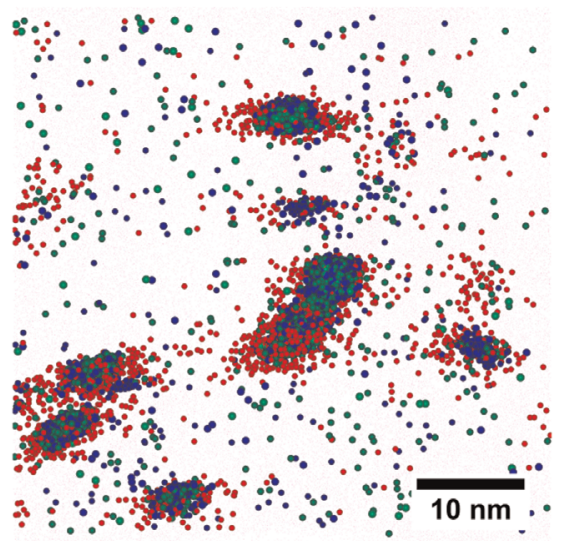

\subsection{Measuring cluster composition}

\subsubsection{Systematic ranging of the mass spectrum}

The result of an APT experiment is a list of 3D atomic positions and an accompanying time-of-flight mass spectrum. In this spectrum peaks at particular mass-to-charge $(\mathrm{m} / z)$ ratios define chemical species and peak identity is determined by the operator. For these alloys, peaks at 1, 2,17 and 18 Da were identified as $\mathrm{H}$ or $\mathrm{OH}$ species present from the chamber and are not counted in the composition. The $16 \mathrm{Da}$ peak has a low concentration in the matrix, and so may come from the chamber or the sample, but is strongly generated by the oxide particles (Fig. 1a) and therefore is identified as ${ }^{16} \mathrm{O}^{+}$. Where peak overlaps exist, the species of greatest global concentration is chosen for the parent range file of each data set. The handling of these overlaps is detailed in the next section.

The evaporating ions have a well-defined mass and charge, meaning the apparent peak width in the $m / z$ spectrum is a result of the evaporation physics and instrumental broadening [17]. Range windows, or ranges, must be defined within which all ions are given a particular identity. The composition measurement depends on the definition and width of these ranges [12].

Ranges are initially defined by hand using the full width at half maximum height because peak shape, width and height vary from peak to peak and between experiments. Hudson et al. showed that the $\mathrm{FWf}(\mathrm{x}) \mathrm{M}$ systematic approach to ranging resulted in a reduction in the variation of composition measurements between data

b

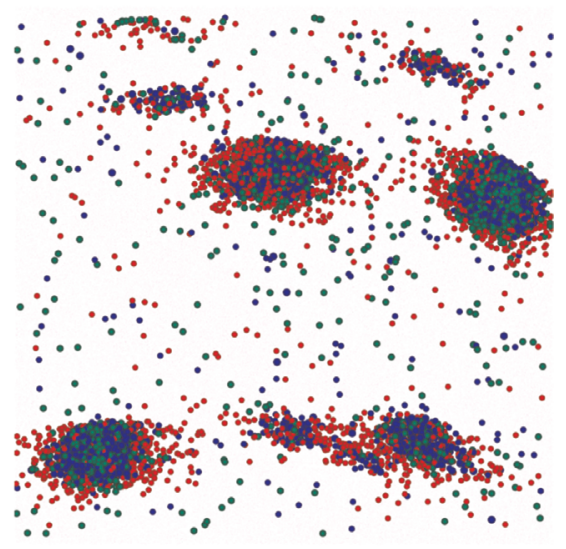

d

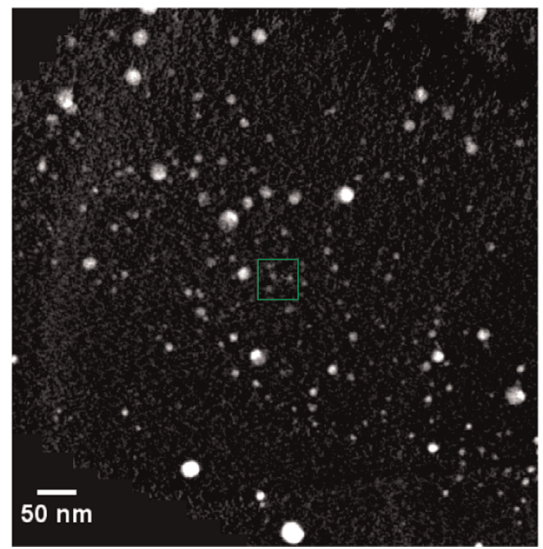

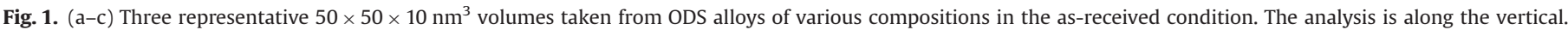

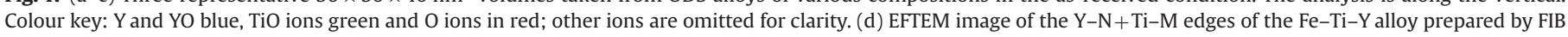

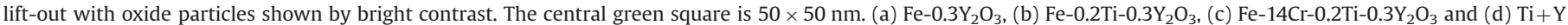
edge EFTEM, Fe- $0.2 \mathrm{Ti}-0.3 \mathrm{Y}_{2} \mathrm{O}_{3}$ (For interpretation of the references to colour in this figure caption, the reader is referred to the web version of this paper.) 
sets [18]. To follow this approach here, a systematic ranging approach has been implemented using a custom MATLAB program to refine the range definitions. Mass peaks are fitted to an exponentially modified Gaussian function [19] to give a smooth peak shape and the full width at $20 \%$ maximum peak height (FW0.2M) was defined following the initial ranging. This value of FW0.2M was chosen to maximise the peak area measured but avoid range overlap due to the narrow separation of some peaks. This custom MATLAB program is highly customisable with respect to the level of $\mathrm{FWf}(\mathrm{x}) \mathrm{M}$ required, definition of peaks and fitting parameters.

\subsubsection{Peak overlaps}

Where peaks overlap in the $m / z$ spectrum, the isotopic ratio of the peaks can be used to separate the contribution of each isobaric ion to a particular peak, called peak deconvolution or decomposition [12]. Conventionally this deconvolution is applied globally across the entire reconstructed volume, but we expect the nature of these overlaps to differ between the matrix and clusters (and indeed between different types of clusters). Given this, there are significant benefits in using a local decomposition approach to carry out accurate chemical characterisation.

In our model ODS alloys, a serious overlap exists between $\mathrm{C}_{2}{ }^{+} /{ }^{48} \mathrm{Ti}^{2+}$, which is avoided by separating the Y-Ti oxide clusters from the matrix because ${ }^{48} \mathrm{Ti}^{2+}$ is only present in the clusters. Beyond separating the clusters from the matrix, different oxide clusters may evaporate with different ionic compositions, meaning ion overlaps must be considered on a cluster-by-cluster basis. An example is the 32 Da peak which contributes roughly $20 \%$ to the total ionic cluster composition (not including $\mathrm{Fe}$ or $\mathrm{Cr}$ ). A variety of complex molecular oxide ions are routinely generated by the oxide clusters, including $\mathrm{O}_{2}{ }^{+}$and $\mathrm{TiO}^{2+}$ ions, both with a $\mathrm{m} / \mathrm{z}$ of approximately $32 \mathrm{Da}$. Depending on the alloy, experimental conditions and original particle chemistry, either $\mathrm{O}_{2}{ }^{+}$or $\mathrm{TiO}^{2+}$ species will predominate in this peak.

To solve these ambiguities another MATLAB script was used to analyse separately the $m / z$ spectrum of each cluster and perform a decomposition. Where the cluster contained too few atoms to make a sufficient $\mathrm{m} / \mathrm{z}$ spectrum, typically fewer than 100 , it was added to a list of small clusters and the total "small clusters" mass spectrum was decomposed at the end of the calculation. A background subtraction using an exponential fitted in time of flight space was applied to each peak.

\section{Results}

The oxides that are observed in the TEM as well-defined particles appear as clusters in the atom probe (point-cloud) data, Fig. 1. Oxygen spreading is observed in the $x-y$ direction, across the surface of the evaporating tip $[9,20]$, but relatively sharp interfaces are seen in the Y and Ti containing ions-especially at the "top" surface of the clusters. We know from TEM that the particles have a spherical or faceted shape, so the shape observed in APT is due to the local variation of tip shape during evaporation and the limitations of the reconstruction protocol.

\subsection{Cluster composition}

After isolating the oxide clusters from the matrix, the apparent Fe content was 60-80 at\%. Evidence from analytical TEM investigations does not show a significant content of Fe in the particles [10], but this measurement is complicated by the ferritic matrix. Studies on chemically extracted particles have shown there to be no Fe present [21]. Williams et al. argued that the inclusion of such a large amount of Fe in the clusters was probably an artifact of the APT experiment and thus applied a matrix correction,

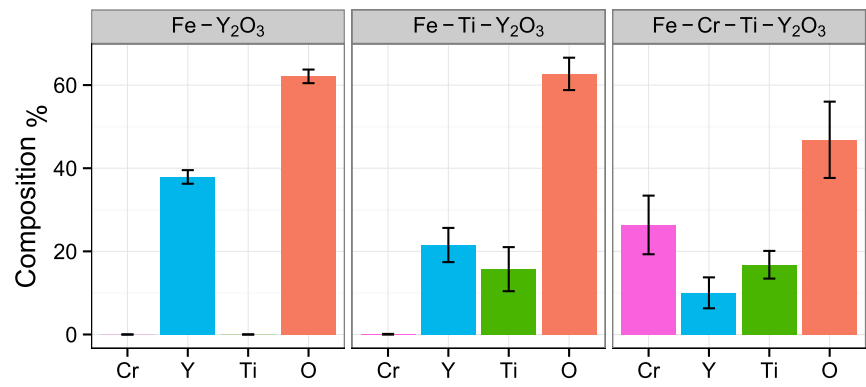

Fig. 2. Matrix-corrected cluster compositions of the three ODS alloys in at\%. The error bars indicate the variation between data sets, not the variation between individual clusters or counting error.

where $\mathrm{Fe}$ and a proportional amount of $\mathrm{Cr}$ are removed from the analysis [22]. Given the evidence from TEM we take the same approach.

The matrix-corrected composition of all the clusters isolated from the matrix and after resolving the identified peak overlaps are shown in Fig. 2. There is variation of composition between data sets as well as a variation in the oxide cluster number density, indicating that these alloys are not homogeneous on the scale observed by APT.

Part of the variation in composition can also be attributed to the composition variation of the individual clusters.

In the Fe-Y alloy the clusters had a $\mathrm{Y}: \mathrm{O}$ ratio of between 0.61 and 0.75 , consistent with the TEM observations which identified $10 \mathrm{~nm}$ cubic- $\mathrm{Y}_{2} \mathrm{O}_{3}$ particles. There is more individual variation in cluster composition in the Ti containing alloys as shown: the Y:Ti: $\mathrm{O}$ ratios of individual clusters in Fig. 3 which are separated into smaller and larger clusters.

For clarity, only a small radius range, $1-1.5 \mathrm{~nm}$, is used to limit the number of points in the left panel of Fig. 3. The arrows show the change in composition after applying the decomposition methodology to each cluster independently, and the circles (227 and 215) indicate the positions of bulk oxide compositions observed by TEM. The majority of smaller clusters show a decrease in $\mathrm{O}$ fraction after decomposition whereas the larger clusters show a uniform increase in oxygen fraction. This correction occurs from the resolution of the peak overlaps $\mathrm{O}_{2}{ }^{+} / \mathrm{TiO}^{2+}$ and $\mathrm{TiO}^{+} / \mathrm{Fe}_{2} \mathrm{O}^{2+}$ at 32 and 64 Da respectively. These overlaps are observed to vary as a function of cluster size, with the smaller clusters producing proportionally more TiO ions than the larger clusters. This effect is most obvious for the large yttrium-poor clusters; most likely $\mathrm{Cr}$ or Ti oxides that evaporate with predominately $\mathrm{O}_{2}{ }^{+}$. After the percluster decomposition, the average $\mathrm{O}$ fractions of both the smaller and larger clusters show much less scatter.

In the Fe-Ti-Y alloy the majority of non-Y/Ti oxygen-containing ions are also Fe-containing, and so many more $\mathrm{Fe}_{2} \mathrm{O}^{2+}$ ions are observed. This results in a correction of about $+10 \%$ in the 0 fraction of the larger $(>3 \mathrm{~nm}$ ) clusters after resolving the $64 \mathrm{Da}$ $\mathrm{TiO}^{+} / \mathrm{Fe}_{2} \mathrm{O}^{2+}$ overlap.

Fig. 2 shows that the $\mathrm{Fe}-\mathrm{Cr}-\mathrm{Ti}-\mathrm{Y}$ alloy oxide clusters apparently contain $26 \pm 7$ at\% $\mathrm{Cr}$ even after applying the matrix correction. The higher metal:oxygen ratio of these $\mathrm{Fe}-\mathrm{Cr}-\mathrm{Ti}-\mathrm{Y}$ alloy clusters indicates either a sub-stoichiometric oxide or the mistaken inclusion of $\mathrm{Cr}$. Investigating the $\mathrm{Cr}$ content of the clusters requires careful consideration of the evaporation process and tip shape during the atom probe experiment and is detailed in the next section.

\subsection{Cluster structure}

No crystallographic information is preserved in the APT data of the oxide clusters but information is available from the 


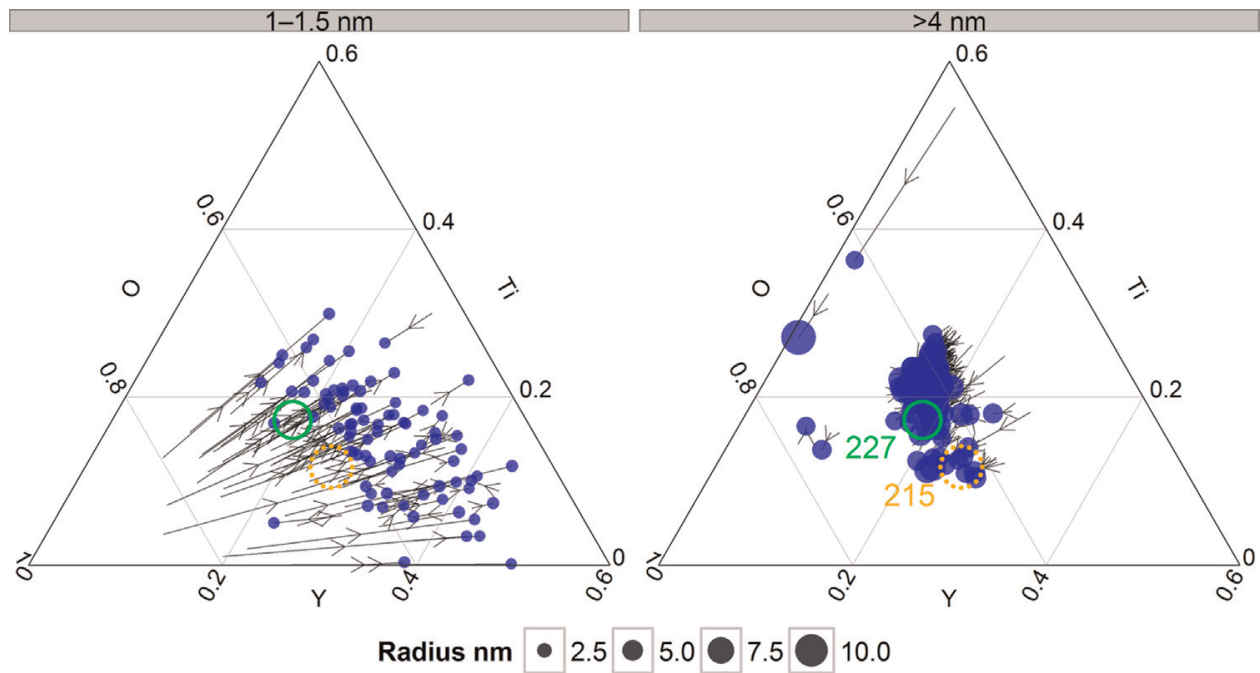

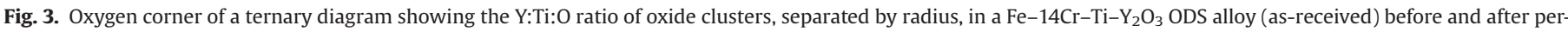

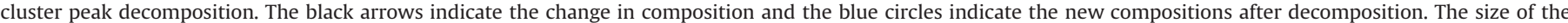

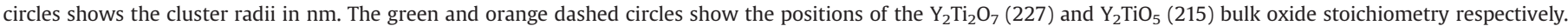
(For interpretation of the references to colour in this figure caption, the reader is referred to the web version of this paper.)

composition and the ionic density surrounding the clusters. Due to the low evaporation field of the oxide particles relative to the matrix [9], the oxides evaporate more readily than the matrix and modify the local tip shape [23]. The modification of the tip shape from a hemisphere and the consequent deviation of ion flight paths by the surface topography are termed trajectory aberrations [23-26].

\subsubsection{Trajectory aberrations around particles}

The low evaporation field of the oxide particles results in incorrect reconstruction of their $Z$ coordinate [27] and deformation of the tip shape. The flattened tip shape causes a demagnification of the clusters, leading to compression of the reconstructed clusters in the $X-Y$ axis [26]. The demagnification is accompanied by the incorporation of ions evaporating from the matrix inside what is considered the particle region of the detector-resulting in unphysically high ionic densities [9].

The average atomic density of Fe is correctly measured as approximately 31 atoms $/ \mathrm{nm}^{3}$ (after accounting for detection efficiency) but variations occur due to the crystallography (zone-lines [28]). Fig. 4a shows the ionic Fe density for the Fe-Cr-Ti-Y alloy in the vicinity of a $<5 \mathrm{~nm}$ oxide cluster (shown by the $\mathrm{Y}$ ions), where the density exceeds 70 ions $/ \mathrm{nm}^{3}$ at the core and drops to 20 "below" the cluster. These results are consistent with the findings of others working on similar materials $[5,29]$.

The extent of any local magnification will be a function of both cluster size and the instantaneous tip radius. Indeed in our data it was observed that the apparent Fe content increased with increasing tip radius. As the electrostatics governing the evaporation process have no inherent scale, the cluster radii can be expressed helpfully as the dimensionless reduced radius, $r^{\prime}=$ cluster radius over the instantaneous tip radius (given by the Geiser et al. method [30]). The Fe content of the clusters was seen to increase with increasing voltage, so using reduced radius removes this complication and allows us to show simply the dependence of included Fe ions as a function of cluster size. Fig. 4c shows how the Fe fraction varies as a function of reduced radius for over 700 oxide clusters in both the $\mathrm{Fe}-\mathrm{Cr}-\mathrm{Ti}-\mathrm{Y}$ and $\mathrm{Fe}-\mathrm{Ti}-\mathrm{Y}$ alloys (black circles).

Part of this dependence comes from the cluster selection method which includes a region of matrix ions surrounding each cluster in the composition. Small statistically random clusters may a

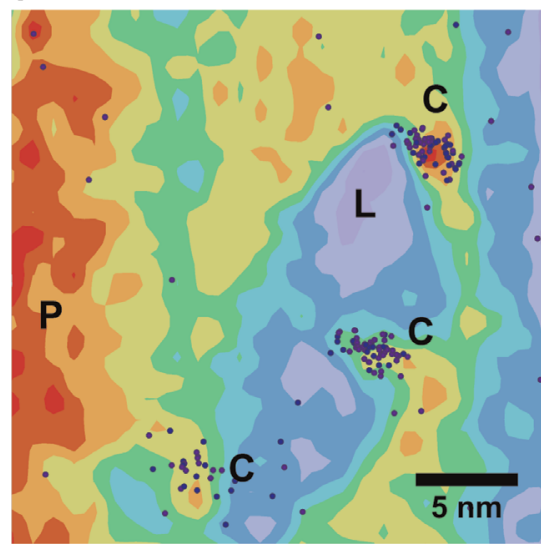

b

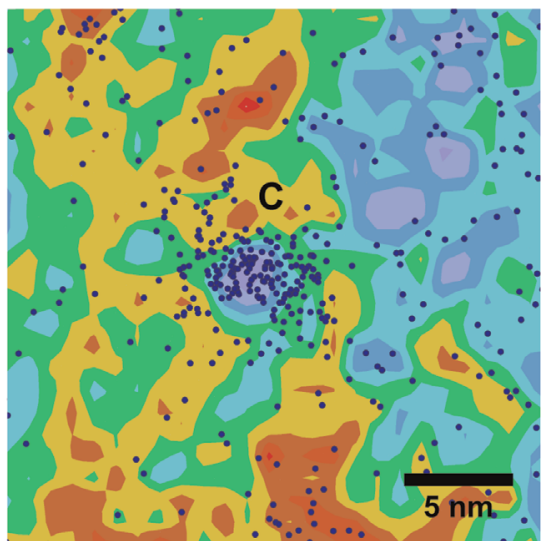

C

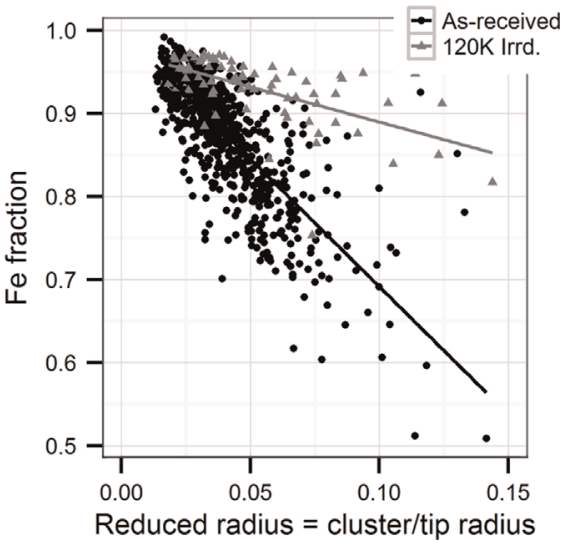

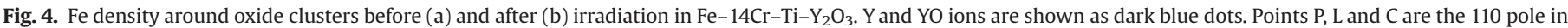

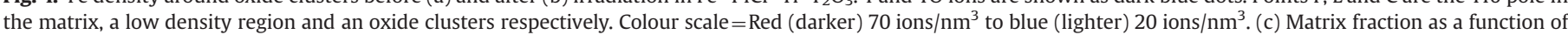

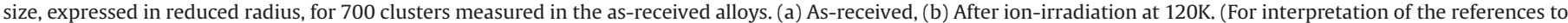
colour in this figure caption, the reader is referred to the web version of this paper.) 

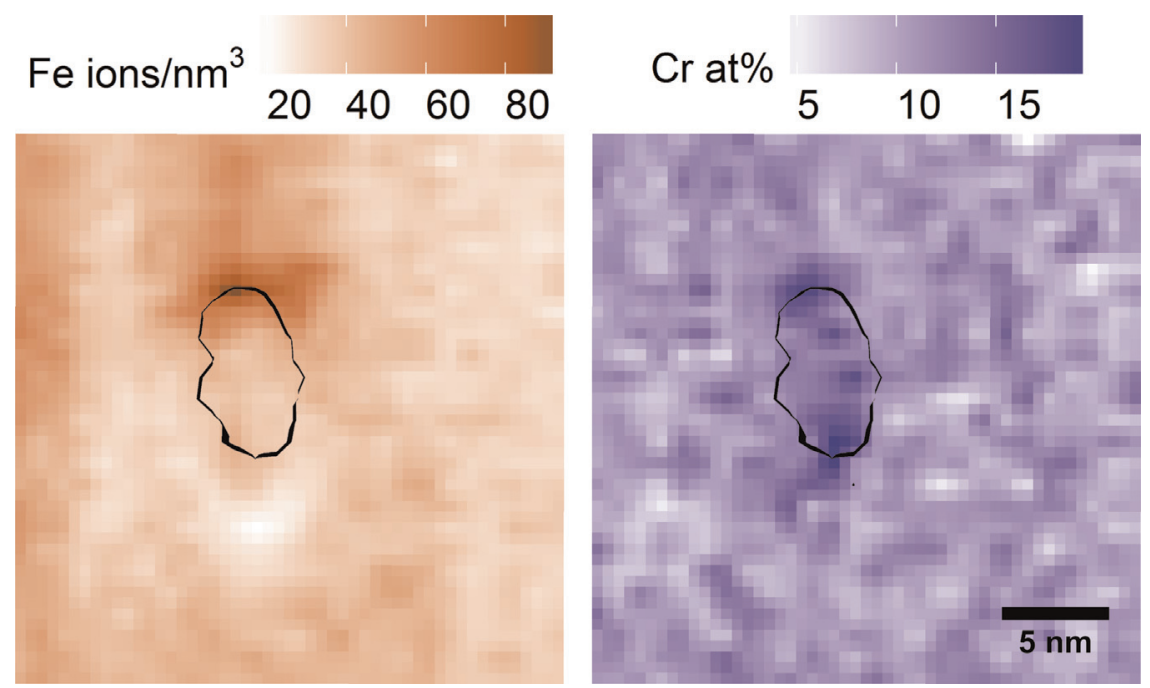

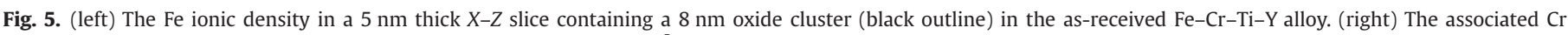

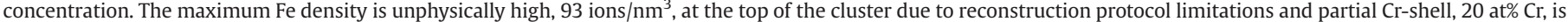
observed at the top and bottom of the cluster. Below the cluster a very low Fe density is observed. The analysis direction is aligned vertically.

also be present which have low solute concentrations [31].

The grey triangles in Fig. 4c also show the clusters observed in $\mathrm{Fe}-\mathrm{Cr}-\mathrm{Ti}-\mathrm{Y}$ after irradiation at $150 \mathrm{~K}$ by self-ions to $100 \mathrm{dpa}$ $\left(9 \times 10^{16}\right.$ ions $\left./ \mathrm{cm}^{2}\right)$. These clusters show a higher Fe content but the structure of the clusters is also dramatically modified, as shown in Fig. 4b. In these clusters, the distribution of solute ions is more spatially diffuse (lower Y-Ti-O density) and the centre of the clusters contains relatively few Fe ions. This suggests that the evaporation behaviour of the clusters reflects something of their atomic structure after irradiation damage.

\subsection{2. $C r$-shells}

Fig. 5 shows the $\mathrm{Cr}$ concentration in a $5 \mathrm{~nm}$ thick $X-Z$ slice through a $8 \mathrm{~nm}$ long oxide cluster in the $\mathrm{Fe}-\mathrm{Cr}-\mathrm{Ti}-\mathrm{Y}$ alloy. Again, an unphysically high Fe density is observed, where the evaporation front initially intersects the oxide cluster and a low density is observed below the cluster. The $\mathrm{Cr}$ concentration at the top is greater than that of the bulk alloy, roughly 20 at\%, compared to 12 at\% in the bulk. The $\mathrm{Cr}$ concentration is also enhanced in the centre and at the bottom of the cluster. The morphology of the cluster is consistent with the reconstruction of a low-field particle: high atomic density and compression in the $X-Y$ plane [26]. Due to the large deformation of the clusters perpendicular to the analysis direction, we propose that concentration line profiles taken along the $Z$ direction will be subject to less distortion. It is not possible to make use of a proximity histogram [32] in this instance because the concentration profile is clearly non-spherical.

Using MATLAB, the reconstructed data and the location of identified clusters, composition line profiles of $1 \times 1 \mathrm{~nm}^{2}$ area and a length scaled to the size of each cluster were measured. Because the profile lengths are normalised, composition plots can be made by averaging all 500 clusters in the $\mathrm{Fe}-\mathrm{Cr}-\mathrm{Ti}-\mathrm{Y}$ alloy, as shown in Fig. 6. In order to address peak overlap issues, a deconvolution process as described in Section 3.1 was used for each cluster assuming that the peak overlaps have the same magnitude for the whole cluster, which may not be valid for composite particles. The line profiles of individual clusters in ODS alloys often show a $\mathrm{Cr}$ rich shell [9] but these profiles are less clear for smaller clusters. Once the clusters are averaged in our data, the $\mathrm{Cr}$-shell becomes more apparent. The profiles in Fig. 6 are elemental, with the exception of the $\mathrm{CrO}$ ions which are shown separately. These $\mathrm{CrO}$ ions do not show a profile similar to that of the core elements or the $\mathrm{Cr}$ shell, suggesting that they are not part of the core or the shell of the cluster but the product of oxygen migration [33] and evaporation with the matrix/shell atoms.

The $\mathrm{Cr}$ shells show a concentration of about 20 at\% with some enrichment in the centre, with the smaller clusters showing more $\mathrm{Cr}$ in the centre. This is presumably due to the trajectory aberrations which overlap the shell with the cluster core [29]. The normalised shell width is the same for each of the cluster size ranges, about 1 cluster extent measured along the analysis direction from which we can infer that the $\mathrm{Cr}$-shell width increases linearly with cluster size. Quantifying the exact shell thickness is difficult, especially on a cluster-by-cluster basis and due to the distortions present, but a geometric approach, such as that by Hatzoglou et al., may be applicable [29].
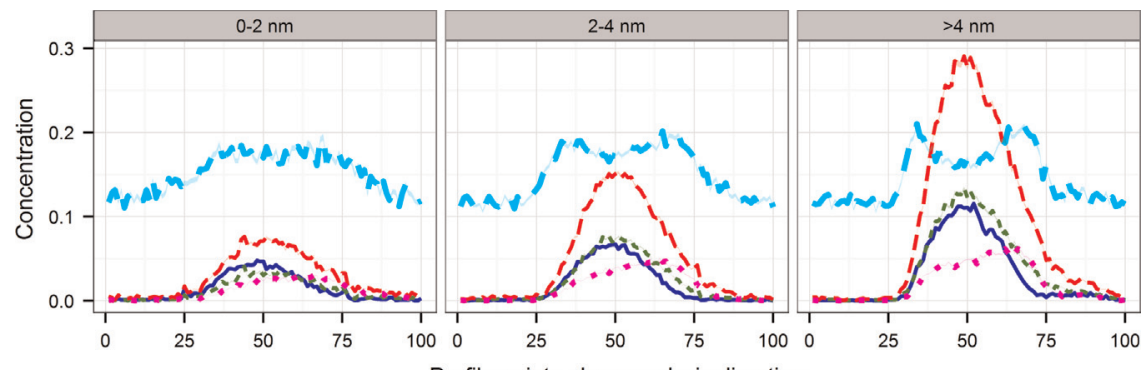

Profile points along analysis direction
$-\mathrm{Y}--\cdot \mathrm{Ti}--\mathrm{O}-\mathrm{Cr} \cdot=\mathrm{CrO}$

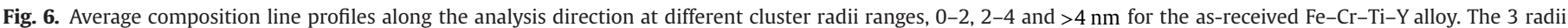

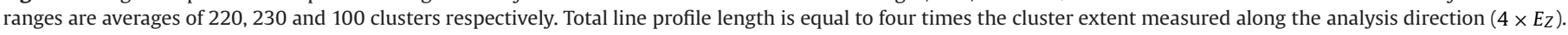


a

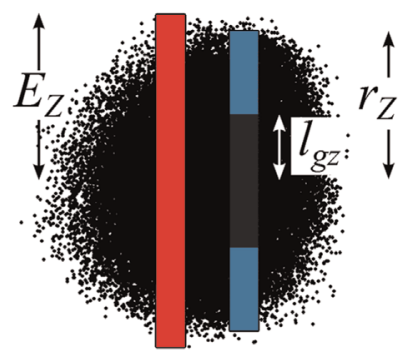

b

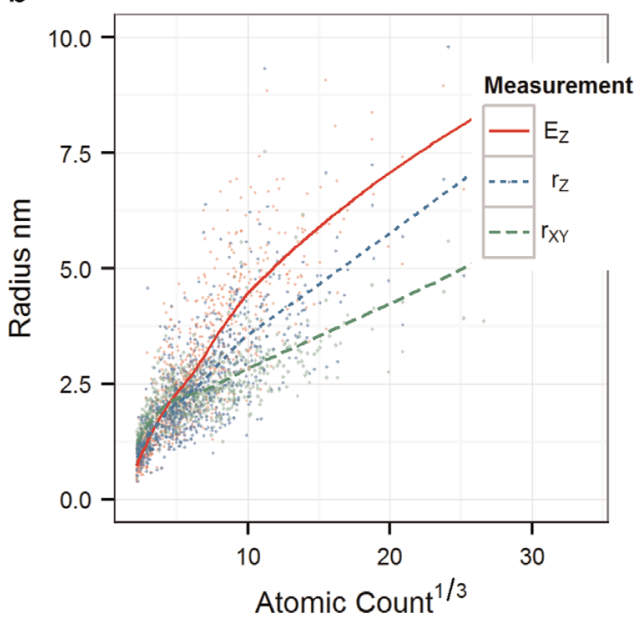

Fig. 7. Comparison of cluster measurement methods. (a) Left: Extent $z$ (red); right: $l_{g z}$ and $r_{Z}$ (blue). (b) Comparison of radii measurements of observed clusters in the as-received alloys against the cube root of atomic count which is proportional to cluster radius. (For interpretation of the references to colour in this figure caption, the reader is referred to the web version of this paper.)

\subsection{Particle size}

Clusters of solute ions are identified using the method of maximum separation and the radius of gyration $l_{g}$ is a convenient way of measuring cluster size [34]. As detailed in Section 3.2.2, we propose that measurement of the clusters should be performed parallel to the analysis direction; the direction least affected by trajectory aberrations [26]. Therefore the $Z$-component of $l_{g}$ and $l_{g z}$ is used. The extent or range $\left(E_{Z}\right)$ and the atomic count are also considered as measurements of cluster size. Atomic count is assumed to be proportional to atomic volume and therefore the cube root of atomic count is proportional to cluster radius. Fig. 7a compares the measurements of $E_{Z}, l_{g z}$ and $r_{Z}\left(r_{Z}=\right.$ corrected $l_{g z}$, that is, $l_{g z} \sqrt{5}$ ) of cluster size along the $Z$ direction of a real cluster. $l_{g z}$ is multiplied by $\sqrt{5}$ to give the approximate hard-sphere radius [34]. The corrected radius of gyration $r_{Z}$ gives an intuitive estimate of the cluster length and $E_{Z}$ gives a slight overestimate due to the spatial blurring of the cluster ions. These measurements are consistent for other clusters and the cluster radii for the $\mathrm{Fe}-\mathrm{Cr}-\mathrm{Ti}-\mathrm{Y}$ and $\mathrm{Fe}-\mathrm{Ti}-\mathrm{Y}$ alloys are plotted against atomic count in Fig. 7b.

The measurement of extent is consistently greater than that of $r_{Z}$ by $20 \%$ for the larger clusters. This is thought to be because of the reduced spatial resolution (Fig. 7a) in the vicinity of the clusters as a result of trajectory aberrations. The $X-Y$ component of the Guinier radius is shown in Fig. $7 \mathrm{~b}$ with comparison to $r_{Z}$ showing the aspect ratio of the clusters as a function of size. It was observed that larger clusters were long and thin (e.g. Fig. 5) and smaller clusters tended to a flatter appearance, such as those in Fig. 1c. These findings are consistent with the evaporation behaviour of relatively low-field clusters with the accompanying aberrations and incorrect $Z$ reconstruction. Following these findings, we shall a

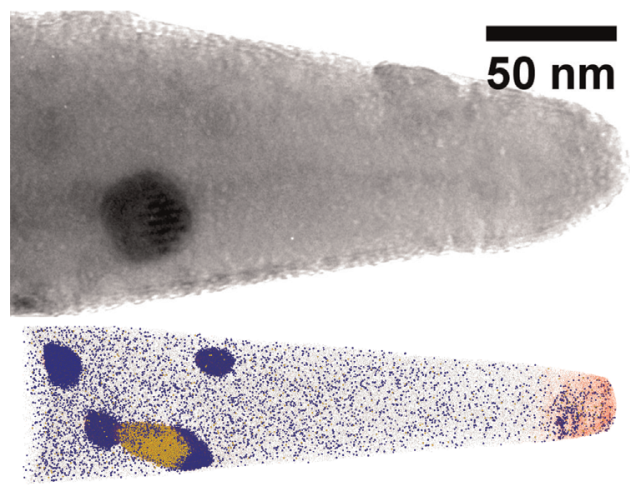

b

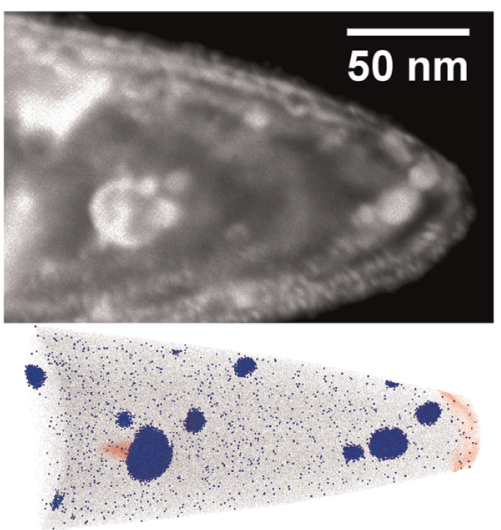

Fig. 8. Correlative microscopy of ODS alloys. In the APT reconstructions (a) and (b), oxide clusters are highlighted by Y-containing ions (blue). $\mathrm{FeO}$ ions (red) indicate the surface oxide on the tips. This APT data was reconstructed using the tip profile image to ensure the global scale is correct. (a) $\mathrm{Fe}-\mathrm{Y}_{2} \mathrm{O}_{3}$ and (b) $\mathrm{Fe}-\mathrm{Ti}-\mathrm{Y}_{2} \mathrm{O}_{3}$. (For interpretation of the references to colour in this figure caption, the reader is referred to the web version of this paper.)

proceed by using the corrected $Z$-component of the radius of gyration $r_{Z}$ as a measure of cluster radius in our APT data.

There are many methods for measuring particle sizes in alloys, but using TEM has the advantage of being able to examine atom probe samples prior to APT analysis-correlative microscopy [35]. Particle sizes measured by TEM are regarded as being directly interpretable, although the image size and contrast mechanisms must be taken into account [36]. Fig. 8 gives two examples of $\mathrm{Fe}-\mathrm{Y}$ (a) and Fe-Ti-Y (b) TEM images before analysis and the accompanying atom maps. Both images contain composite particles: in (a) the larger particle shows Moiré fringes in TEM and is composed of a $\mathrm{Cr}$ oxide attached to a Y oxide; in (b), the larger particle is revealed by APT to be half Y oxide and half Fe oxide. Reconstructions were performed using the tip profile method in IVAS [37] and inconsistencies in tip shape due to the clusters were accounted for by modifying the tip radius profile [38].

The smaller average particle size in the $\mathrm{Fe}-\mathrm{Cr}-\mathrm{Ti}-\mathrm{Y}$ alloy made matching TEM images to APT reconstructions difficult, but the same reconstruction procedure was applied and the resulting particle size distribution is shown later in Fig. 10.

\section{Discussion}

\subsection{Error analysis}

The greatest contribution to the error in the compositional measurement comes from misidentification of peaks or peak 


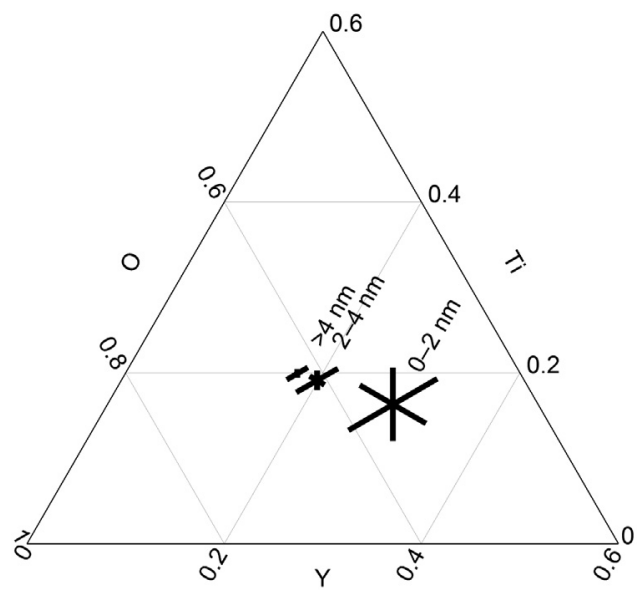

Fig. 9. Compositional error due to counting error of $0-2,2-4$ and $>4 \mathrm{~nm}$ Y-Ti-O clusters in $\mathrm{Fe}-\mathrm{Cr}-\mathrm{Ti}-\mathrm{Y}$ ODS steel. The error bar positions show the average composition of the three size ranges of clusters after a per-cluster deconvolution of overlapping mass peaks. Average cluster size: 55, 500 and 2000 ions.

overlaps. For example, the 24 Da peak can be ranged as $\mathrm{C}_{2}{ }^{+}$, which is appropriate for the matrix, but is predominately ${ }^{48} \mathrm{Ti}^{2+}$ in the clusters. Either of these identifications are correct but each is applicable in different circumstances. This identification led to a $25 \%$ difference in the total concentration of Ti detected in the clusters. A cautious approach is required in range identification to minimise human error and misidentification. Where the origin of overlapping species is different in different volumes, the local deconvolution method (Section 2.1.2) will give a better interpretation of the composition. The non-global composition approach presented here is not only applicable to clusters but could also be employed in the study of interfaces or multiple-phase reconstructions where the peak overlaps are not globally consistent.

Peak deconvolution partially relaxes the constraint on the identification of ions, but introduces some contribution to compositional error. This error arises from the uncertainty of extrapolating the least-squares fitting used to calculate the extent of the peak overlaps and is influenced by the difference in tabulated and actual isotopic abundances, range width and $\mathrm{m} / \mathrm{z}$ spectrum noise. The least-squares fitting is robust to noise, and the peak counts are background corrected, but there is some small error associated with this and the background noise estimation is not perfect.

The next significant source of compositional error comes from the choice of range width. To estimate this, three range files were created: one by intuitively ranging most of the peak, one by ranging from background to background, and a MATLAB-optimised FW0.2M range file. The findings were consistent with [18], with reduced reliability of measurement with decreasing alloy content. The errors were $\mathrm{Fe} 0.1 \%, \mathrm{Cr} 1 \%$ and $5-10 \%$ for $\mathrm{Y}$, Ti and $\mathrm{O}$. These are not counting errors, which for even the lowest concentration solute is only $0.8 \%$.

The systematic ranging approach, Hudson et al. [18] used, relies on the assumption that $\mathrm{FWf}(\mathrm{x}) \mathrm{M}$ is proportional to the peak area in $m / z$ space across the whole mass spectrum. The practicality of this assumption is that range files must be generated on a per-data set basis, as the peak area varies with peak shape and amplitude from data set to data set. Johnson [17] showed that the asymmetry of the peaks can be significant and that for an accurate measure of peak area the peak tails must be accounted for. This is acceptable where there are sufficient $m / z$ counts to form a smooth spectrum but does not resolve the issues of individual ion identity required for the cluster selection. Ultimately a compromise of accuracy and computational difficulty must be considered given the relatively small significance of the choice of range width on composition.

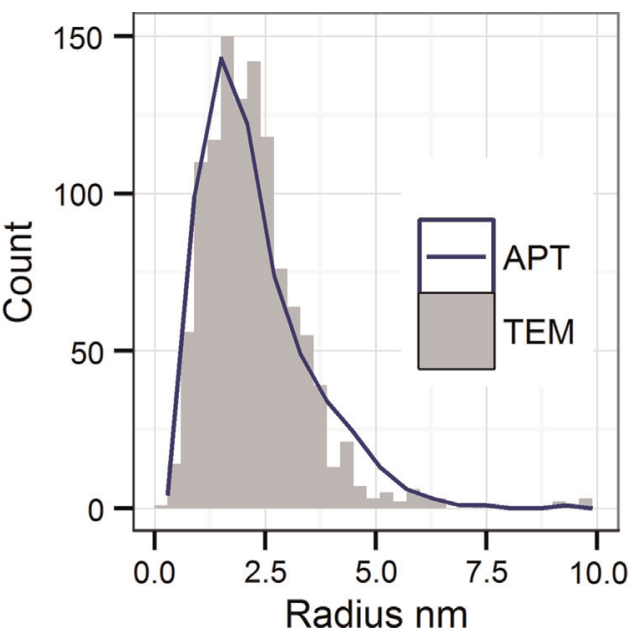

Fig. 10. Indirectly correlated data obtained by TEM (radius of $>1000$ particles) and APT ( $r_{Z}$ of $>500$ clusters) measurements of the particle size distribution in the $\mathrm{Fe}-\mathrm{Cr}-\mathrm{Ti}-\mathrm{Y}_{2} \mathrm{O}_{3}$ alloy.

Due to the $37 \%$ detector efficiency of the LEAP system, statistical counting error becomes significant for small samples, such as sub-1 $\mathrm{nm}$ oxide clusters. The average counting error associated with the number of atoms detected from oxide clusters in the $\mathrm{Fe}-$ $\mathrm{Cr}-\mathrm{Y}-\mathrm{Ti}$ alloy is shown in Fig. 9. For the $0-2 \mathrm{~nm}$ clusters, the median number of detected ions was only 55. Even with the counting error, the mean positions of the differently sized clusters show a progression to a higher $\mathrm{O}$ fraction and decreased $\mathrm{Y}$ :Ti ratio.

\subsection{Implications for ODS analysis}

Others have noted the variation of cluster compositions of ODS alloys as measured by APT [5,22]. Figs. 3 and 9 show a variation in cluster composition in excess of the errors associated with the measurement (Section 4.1). For the larger oxides, shown in right panel of Fig. 3, a range of Y:Ti ratios is observed, some consistent with the bulk stoichiometries of $\mathrm{Y}_{2} \mathrm{Ti}_{2} \mathrm{O}_{7}$ and $\mathrm{Y}_{2} \mathrm{TiO}_{5}$, but others with $\mathrm{Y}$ : $\mathrm{Ti}<1$. This high Ti content has been attributed by some to $\mathrm{Ti}$ segregation to the cluster interface [39], but we found no evidence of this in the cluster composition line profiles. Structures consistent with $\mathrm{Y}_{2} \mathrm{Ti}_{2} \mathrm{O}_{7}$ and $\mathrm{Y}_{2} \mathrm{TiO}_{5}$ were observed by TEM but this does not necessarily mean that particles with these exact compositions are present. The high surface area-to-volume ratio, the presence of the Cr-shell or a defective structure could all account for a deviation from the expected stoichiometry [40]. We have not noticed any preferential ion loss due to multiple hits [20], but the influence of laser wavelength has not been investigated and could explain the oxygen deficiency of the larger clusters [41].

As for the size measurement of the particles, with the correct global reconstruction scale, the $Z$ component of the Guinier radius $\left(l_{g z} \times \sqrt{5}\right)$ gives good agreement with the size measurement from TEM as shown in Fig. 10. This figure shows the particle size measurement of indirectly correlated microscopy (TEM and APT of different regions), informed by directly correlative microscopy.

One aspect that has not been addressed here is the limited sample volume of APT and the inhomogeneity common to many ODS and powder-processed alloys [6]. Fig. 1d shows a range of particle sizes and variation in local particle density. The cluster number density measured by APT for $\mathrm{Fe}-\mathrm{Cr}-\mathrm{Ti}-\mathrm{Y}$ ranged from 0.20 to $5.6 \times 10^{23} \mathrm{~m}^{-3}$ between sample regions (with an approximate counting error of only $0.3 \times 10^{23} \mathrm{~m}^{-3}$ ). The inhomogeneity of the material may have an influence on the mechanical properties; very large oxides can reduce fatigue life [42] and the 3D distribution of particles is used to inform dislocation dynamics models [43]. 


\section{Conclusion}

Consistent measurements of cluster composition over many data sets have been demonstrated using systematic ranging and a new region-specific peak deconvolution method. The findings of field evaporation simulations and correlative microscopy have informed the choice of cluster radius measurement to give very good agreement between TEM and APT data, even on differing sample areas. A Cr-shell ( 20 at\% $\mathrm{Cr}$ ) in the $\mathrm{Fe}-\mathrm{Cr}-\mathrm{Ti}-\mathrm{Y}$ alloy was reliably observed, even in the presence of significant trajectory aberrations, by averaging composition profiles along the analysis direction. The Cr-shell width increased linearly with cluster size, suggesting either segregation of $\mathrm{Cr}$ to the cluster surface [22] or displacement of the $\mathrm{Cr}$ from the cluster during processing. The aberrations present were quantified in terms of the Fe ionic density and were shown to be a function of composition and oxide structure by comparison with ion-irradiated samples. By careful consideration of the error, we justified the measurements of the Y: Ti ratio of the Y-Ti-O clusters with size. Ranging the low alloy content $(<0.3$ at $\%)$ components was the least reliable process and counting error dominated for the sub $1 \mathrm{~nm}$ radius, $<100$ ion, clusters.

\section{Acknowledgements}

The authors thank Dr. Tom Black for funding part of this project and EPSRC for support under Grant number EP/I012400/1; Prof. A. Kirkland and Dr. J. Kim for the support on the 2200 microscope.

\section{References}

[1] G.R. Odette, M.J. Alinger, B.D. Wirth, Recent developments in irradiation-resistant steels, Annu. Rev. Mater. Res. 38 (2008) 471-503.

[2] M.K. Miller, C.M. Parish, Q. Li, Advanced oxide dispersion strengthened and nanostructured ferritic alloys, Mater. Sci. Technol. 29 (10) (2013) 1174-1178.

[3] D.T. Hoelzer, J. Bentley, M.A. Sokolov, M.K. Miller, G.R. Odette, M.J. Alinger, Influence of particle dispersions on the high-temperature strength of ferritic alloys, J. Nucl. Mater. 367 (2007) 166-172.

[4] L.L. Hsiung, M.J. Fluss, S.J. Tumey, B.W. Choi, Y. Serruys, F. Willaime, A. Kimura, Formation mechanism and the role of nanoparticles in $\mathrm{Fe}-\mathrm{Cr}$ ods steels developed for radiation tolerance, Phys. Rev. B 82 (2010) 184103.

[5] M.K. Miller, E.A. Kenik, K.F. Russell, L. Heatherly, D.T. Hoelzer, P.J. Maziasz Atom probe tomography of nanoscale particles in ods ferritic alloys, Mater. Sci. Eng.: A 353 (1) (2003) 140-145.

[6] A.G. Certain, K.G. Field, T.R. Allen, M.K. Miller, J. Bentley, J.T. Busby, Response of nanoclusters in a $9 \mathrm{Cr}$ ODS steel to $1 \mathrm{dpa}, 525^{\circ} \mathrm{C}$ proton irradiation, J. Nucl. Mater. 407 (1) (2010) 2-9.

[7] I.S. Kim, J.D. Hunn, N. Hashimoto, D.L. Larson, P.J. Maziasz, K. Miyahara, E H. Lee, Defect and void evolution in oxide dispersion strengthened ferritic steels under $3.2 \mathrm{mev} \mathrm{Fe}+$ ion irradiation with simultaneous helium injection, J. Nucl. Mater. 280 (3) (2000) 264-274.

[8] D.J. Larson, P.J. Maziasz, I.S. Kim, K. Miyahara, Three-dimensional atom probe observation of nanoscale titanium-oxygen clustering in an oxide-dispersionstrengthened $\mathrm{Fe}-12 \mathrm{Cr}-3 \mathrm{~W}-0.4 \mathrm{Ti}+\mathrm{Y}_{2} \mathrm{O}_{3}$ ferritic alloy, Scripta Mater. 44 (2) (2001) 359-364.

[9] E.A. Marquis, Core/shell structures of oxygen-rich nanofeatures in oxide-dis persion strengthened Fe-Cr alloys, Appl. Phys. Lett. 93 (18) (2008) 181904.

[10] M. Klimenkov, R. Lindau, A. Möslang, New insights into the structure of ODS particles in the ODS-Eurofer alloy, J. Nucl. Mater. 386 (2009) 553-556.

[11] G.R. Odette, N.J. Cunningham, Y. Yu, A. Etienne, E. Haney, T. Yamamoto, Multiple technique characterization of a nanostructured ferritic alloy: heat-toheat, long term high temperature thermal aging and friction stir welding effects on nano-scale dispersion strengthening features, Microsc. Microanal. 16 (S2) (2010) 1600-1601.

[12] M.K. Miller, Atom Probe Tomography: Analysis at the Atomic Level, Plenum Pub Corp, New York, 2000.

[13] K. Thompson, D. Lawrence, D.J. Larson, J.D. Olson, T.F. Kelly, B. Gorman, In situ site-specific specimen preparation for atom probe tomography, Ultramicroscopy 107 (2) (2007) 131-139.

[14] S. Lozano-Perez, V. de Castro Bernal, R.J. Nicholls, Achieving sub-nanometre particle mapping with energy-filtered TEM, Ultramicroscopy 109 (10) (2009) $1217-1228$.

[15] J.M. Hyde, C.A. English, Microstructural processing irradiated materials, in: MRS 2000 Fall Meeting, Symposium R, vol. 650, 2001, pp. 27-30.

[16] C.A. Williams, D. Haley, E.A. Marquis, G.D.W. Smith, M.P. Moody, Defining clusters in apt reconstructions of ODS steels, Ultramicroscopy 132 (2013) 271-278.

[17] L.J.S. Johnson, M. Thuvander, K. Stiller, M. Odén, L. Hultman, Blind deconvolution of time-of-flight mass spectra from atom probe tomography, Ultramicroscopy 132 (2013) 60-64.

[18] D. Hudson, G.D.W. Smith, B. Gault, Optimisation of mass ranging for atom probe microanalysis and application to the corrosion processes in $\mathrm{Zr}$ alloys, Ultramicroscopy 111 (6) (2011) 480-486.

[19] P.J. Naish, S. Hartwell, Exponentially modified gaussian functions-a good model for chromatographic peaks in isocratic HPLC? Chromatographia 26 (1) (1988) 285-296.

[20] C.A. Williams, G.D.W. Smith, E.A. Marquis, Quantifying the composition of yttrium and oxygen rich nanoparticles in oxide dispersion strengthened steels, Ultramicroscopy 125 (2013) 10-17.

[21] Y. Wu, E.M. Haney, N.J. Cunningham, G.R. Odette, Transmission electron microscopy characterization of the nanofeatures in nanostructured ferritic alloy MA957, Acta Mater. 60 (8) (2012) 3456-3468.

22] C.A. Williams, E.A. Marquis, A. Cerezo, G.D.W. Smith, Nanoscale characterisation of ods-eurofer 97 steel: an atom-probe tomography study, J. Nucl. Mater. 400 (1) (2010) 37-45.

[23] F. Vurpillot, A. Bostel, D. Blavette, Trajectory overlaps and local magnification in three-dimensional atom probe, Appl. Phys. Lett. 76 (21) (2000) 3127-3129.

[24] F. Vurpillot, D. Larson, A. Cerezo, Improvement of multilayer analyses with a three-dimensional atom probe, Surf. Interface Anal. 36 (5-6) (2004) 552-558.

25] E.A. Marquis, F. Vurpillot, Chromatic aberrations in the field evaporation behavior of small precipitates, Microsc. Microanal. 14 (06) (2008) 561-570.

[26] C. Oberdorfer, G. Schmitz, On the field evaporation behavior of dielectric materials in three-dimensional atom probe: a numeric simulation, Microsc Microanal. 17 (1) (2010) 15-25.

[27] T. Boll, T. Al-Kassab, Yong Yuan, Z.G. Liu, Investigation of the site occupation of atoms in pure and doped tial/ti3al intermetallic, Ultramicroscopy 107 (9) (2007) 796-801.

[28] F. Vurpillot, A. Bostel, D. Blavette, A new approach to the interpretation of atom probe field-ion microscopy images, Ultramicroscopy 89 (1) (2001) 137-144.

[29] C. Hatzoglou, B. Radiguet, P. Pareige, Experimental artifacts occurring during atom probe tomography analysis of ODS steels, in: The 20th Fe-Cr WorkshopRouen, France, 9-10 May 2012, 2012.

[30] B.P. Geiser, D.J. Larson, E. Oltman, S. Gerstl, D. Reinhard, T.F. Kelly, T.J. Prosa, Wide-field-of-view atom probe reconstruction, Microsc. Microanal. 15 (S2) (2009) 292-293.

[31] P.D. Styman, J.M. Hyde, K. Wilford, G.D.W. Smith, Quantitative methods for the apt analysis of thermally aged rpv steels, Ultramicroscopy 132 (2013) 258-264.

[32] O.C. Hellman, J.A. Vandenbroucke, J. Rüsing, D. Isheim, D.N. Seidman, Analysis of three-dimensional atom-probe data by the proximity histogram, Microsc. Microanal. 6 (05) (2000) 437-444.

[33] B. Gault, F. Danoix, K. Hoummada, D. Mangelinck, H. Leitner, Impact of directional walk on atom probe microanalysis, Ultramicroscopy 113 (2012) 182-191.

[34] M.K. Miller, A. Cerezo, M.G. Hetherington, G.D.W. Smith, Atom Probe Field Ion Microscopy, Clarendon Press Oxford, UK, 1996.

35] I. Arslan, E.A. Marquis, M. Homer, M.A. Hekmaty, N.C. Bartelt, Towards bette 3-D reconstructions by combining electron tomography and atom-probe tomography, Ultramicroscopy 108 (12) (2008) 1579-1585.

[36] D.B. Williams, C.B. Carter, The Transmission Electron Microscope, Springer, New York, 1996.

[37] D.J. Larson, T.J. Prosa, R.M. Ulfig, B.P. Geiser, T.F. Kelly, Local Electrode Atom Probe Tomography: A User's Guide, Springer-Verlag, New York, 2013.

[38] D.J. Larson, B.P. Geiser, T.J. Prosa, T.F. Kelly, On the use of simulated field evaporated specimen apex shapes in atom probe tomography data reconstruction, Microsc. Microanal. 18 (5) (2012) 953.

[39] P. Wells, N. Cunningham, G.R. Odette, Recent Progress on Understanding and Quantifying Atom Probe Tomography Artifacts for High Evaporation Rate nmScale Phases in Fe based alloys, 2012.

[40] A. Hirata, T. Fujita, Y.R. Wen, J.H. Schneibel, C.T. Liu, M.W. Chen, Atomic structure of nanoclusters in oxide-dispersion-strengthened steels, Nat. Mater. 10 (12) (2011) 922-926.

[41] M. Bachhav, R. Danoix, F. Danoix, B. Hannoyer, S. Ogale, F. Vurpillot, Investigation of wustite ( $\mathrm{Fe} 1-\mathrm{Xo})$ by femtosecond laser assisted atom probe tomography, Ultramicroscopy 111 (6) (2011) 584-588.

[42] I. Kubena, B. Fournier, T. Kruml, Effect of microstructure on low cycle fatigue properties of ODS steels, J. Nucl. Mater. 424 (1) (2012) 101-108.

[43] B. Bakó, D. Weygand, M. Samaras, W. Hoffelner, M. Zaiser, Dislocation depinning transition in a dispersion-strengthened steel, Phys. Rev. B 78 (14) (2008) 144104. 